\title{
Conductance modulations in spin field-effect transistors under finite bias voltages
}

\author{
Liangbin Hu, Ju Gao, and Shun-Qing Shen \\ Department of Physics, The University of Hong Kong, Pokfulam Road, Hong Kong, China
}

(Received 16 October 2003; published 6 April 2004)

\begin{abstract}
The conductance modulations in spin field-effect transistors under finite bias voltages were studied. It was shown that when a finite bias voltage is applied between two terminals of a spin field-effect transistor, the spin precession states of injected spin-polarized electrons in the semiconductor channel of the device will depend not only on the gate-voltage controlled Rashba spin-orbit coupling but also on the bias voltage and, hence, the conductance modulation in the device due to Rashba spin-orbit coupling may also depend sensitively on the bias voltage.
\end{abstract}

DOI: 10.1103/PhysRevB.69.165304

PACS number(s): 73.40.Sx, 72.25.Hg, 71.70.Ej, 73.21.-b

\section{INTRODUCTION}

In the recent years spin-polarized transport in semiconductor microstructures has attracted much attention because of its important relevance to the emerging field of spintronics, a new branch of electronics where the electron's spin ( in addition to its charge) is the active element for information storage and processing. ${ }^{1} \mathrm{An}$ issue of fundamental importance in the emerging field of spintronics is the generation and control of high spin-polarized currents in semiconductors. ${ }^{1-5}$ Recently high efficient injection of spin-polarized currents from magnetic to nonmagnetic semiconductors have been achieved at low temperatures; ${ }^{6}$ however, efficient injection of spin-polarized currents from ferromagnetic $(F)$ metals into semiconductors $(S)$ has not yet been realized experimentally. But for room temperature spintronic devices, ferromagnetic metal sources are indispensable tools. Detailed theoretical investigations have revealed that the main obstacle for spin injection from an $F$ metal source into a semiconductor originates from the large mismatch between the conductivities of metals and semiconductors. ${ }^{7,8}$ It can be shown that in usual $F S$ junctions, the spin injection coefficients are proportional to $\sigma_{S} / \sigma_{F}$, where $\sigma_{S}$ and $\sigma_{F}$ are the conductivities of the semiconductors and the $F$ metals, respectively. Since $\sigma_{S}$ $\ll \sigma_{F}$, the efficiencies of spin injections in usual $F S$-junctions are very small. At first glance, this problem seems insurmountable, but very recent theoretical investigations show that this obstacle may be overcome through the use of suitable potential barriers ${ }^{8-10}$ or through appropriate epitaxial interfaces that obey certain selection rules and band-structure symmetry properties, ${ }^{11,12}$ and encouraging experimental results have also been obtained following the theoretical predictions. ${ }^{13-15}$ These results suggest that devices made of combinations of $F$ metals and semiconductors may be truly promising for applications in spintronics. Among the most prominent device proposals that involve combinations of $F$ metals and semiconductors is the spin field-effect transistor (spin FET) (Ref. 4). In a spin FET, two ferromagnetic metallic electrodes are coupled via a ballistic semiconductor channel. The current modulation in the structure arises from spin precession of injected spin-polarized electrons in the semiconductor channel due to Rashba spin-orbit coupling, while two ferromagnetic metallic electrodes are used to preferentially inject and detect the spin-polarized currents. It has long been established both theoretically ${ }^{16,17}$ and experimentally ${ }^{18,19}$ that, arising from the structural inversion asymmetry, there is a spin-orbit interaction in twodimensional electron gases (2DEG's) on narrow-gap semiconductor (such as InAs) surfaces. This underlying spin-orbit interaction was known as Rashba spin-orbit coupling in the literatures. An important feature of Rashba spin-orbit coupling is that its strength can by tuned by an external gate voltage, which alters the build-in structural inversion asymmetry. Due to this fact, spin precession of injected spinpolarized electrons in the $S$ channel of a spin FET can be tuned by applying an external gate voltage, and concomitantly, the current flowing through the device can be also modulated. This mechanism was first proposed in a seminal work by Datta and Das ${ }^{4}$ and recently, some important factors that will affect the behaviors of a spin FET were investigated in more details and with more realistic assumptions. ${ }^{20-25}$ In the present paper, we discuss the conductance modulations in spin FET's under finite bias voltages. Previous theoretical investigations have been focussed on the zero-bias conductance modulations in spin FET's, but in practical applications a finite bias voltage need to be applied between two terminals of a spin FET, and the conductance-bias voltage characteristics of a device are usually very important for its practical applications. From theoretical viewpoints, when a finite bias voltage is applied between two terminals of a spin FET, a longitudinal electric field will be established in the semiconductor channel of the device, and as was well known, in spin-orbit coupled systems external electric field may play a more subtle role on electron's transport than in traditional electronic devices. The reason for this is that in spin-orbit coupled systems the effect of electric field may be sensitively spin dependent. (Examples of unusual effect of electric field on electron's charge and spin transport in spin-orbit coupled systems can be seen from Refs. 26-28.) In the present paper we discuss the influence of finite bias voltages on the conductance modulations in spin FET's due to Rashba spin-orbit coupling. We will show that when a finite bias voltage is applied between two terminals of a spin FET, the conductance modulation in the structure due to Rashba spin-orbit coupling may depend sensitively on the bias voltage, and in order to describe correctly the spin precession states of injected spin-polarized electrons in the semiconductor channel of the device, the interplay between the Rashba spin-orbit 
coupling (which can be tuned via the gate voltage) and the longitudinal electric field induced by the application of a finite bias voltage should be described in a unified way.

\section{MODEL AND FORMULATION}

For simplicity, in this paper we will restrict our discussion to a 1D model. In one-dimensional systems the quantum interference effect due to Rashba spin-orbit coupling will be maximum since the phase shifts of electrons due to Rashba spin-orbit coupling are independent of their paths, so the idealized 1D model will give an upper limit for the achievable spin-transistor effect. In higher dimensions, the phase shifts of electrons will depend on their paths and, hence, the spin-transistor effect will become weaker than what is predicted in a 1D model system. This was illustrated in Ref. 21. Though in the present paper we restrict our discussion to a 1D model system, the formulas given below are easy to be extended to systems with higher dimensions. This will be discussed elsewhere. In the one-band effective-mass approximation, the $1 \mathrm{D}$ model system can be described by the following Hamiltonian:

$$
\begin{aligned}
\hat{H}= & \frac{1}{2} \hat{p}_{x} \frac{1}{m(x)} \hat{p}_{x}+\frac{1}{2 \hbar} \hat{\sigma}_{z}\left[\hat{p}_{x} \alpha(x)+\alpha(x) \hat{p}_{x}\right] \\
& +\frac{1}{2} \Delta \hat{\boldsymbol{\sigma}} \cdot\left[\vec{m}_{L} \theta(-x)+\vec{m}_{R} \theta(x-L)\right]+\delta E_{c} \\
& \times \theta(x) \theta(L-x)+\hat{U}[\delta(x)+\delta(x-L)]+V(x) .
\end{aligned}
$$

Here $\theta(x)$ is the usual step function and $\delta(x)$ the usual $\delta$ function, $\hat{p}_{x}$ is the momentum operator, $\hat{\boldsymbol{\sigma}}$ is the Pauli matrix, $m(x)=m_{f}+\left(m_{s}-m_{f}\right) \theta(x) \theta(L-x)$ is the effective mass of electron, with $m_{f}$ denoting the effective mass of electron in the ferromagnetic electrodes and $m_{s}$ the effective mass of electron in the semiconductor channel, and the $F / S$ interfaces are assumed to be located at $x=0$ and $x=L$. The second term in Eq. (1) describes the Rashba spin-orbit coupling, ${ }^{21-24}$ where $\alpha(x)$ is defined by $\alpha(x) \equiv \alpha_{R} \theta(x) \theta(L$ $-x$ ), with $\alpha_{R}$ denoting the Rashba spin-orbit coupling constant in the $S$ region, which can be tuned by the gate voltage. Since the Hamiltonian $\hat{H}$ has to be an Hermitian operator, in Eq. (1) we have used the symmetrized version of Rashba spin-orbit interaction. The third term in Eq. (1) describes the exchange interaction in the ferromagnetic electrodes, with $\Delta$ denoting the spin-splitting energy and the unit vector $\vec{m}_{L}$ $\left(\vec{m}_{R}\right)$ denoting the direction of the magnetization in the left (right) electrode. It will be assumed that $\vec{m}_{L}$ is in the $+x$ direction and $\vec{m}_{R}$ will be in either $+x$ direction (parallel configuration) or $-x$ direction (antiparallel configuration). The fourth and fifth terms in Eq. (1) model the conductionband mismatch and the interfacial scattering between the $F$ and $S$ regions, respectively, with $\delta E_{c}$ denoting the band mismatch and $\hat{U}$ the interfacial scattering potential. In the presence of both spin-conserving and spin-flip interfacial scattering, $\hat{U}$ will be a $2 \times 2$ matrix with the diagonal elements $\left(U^{\uparrow \uparrow}, U^{\downarrow \downarrow}\right)$ representing the spin-dependent strength of spin- conserving interfacial scattering and the off-diagonal elements $\left(U^{\uparrow \downarrow}, U^{\downarrow \uparrow}\right)$ the strength of spin-flip interfacial scattering. For simplicity, we will assume that $U^{\uparrow \uparrow}=U^{\downarrow \downarrow} \equiv U_{1}$ and $U^{\uparrow \downarrow}=U^{\downarrow \uparrow} \equiv U_{2}$. (For magnetically active interface, it is possible that $U^{\uparrow \uparrow} \neq U^{\downarrow \downarrow}$ and $U^{\uparrow \downarrow} \neq U^{\downarrow \uparrow}$.) Finally, the last term in Eq. (1) denotes the longitudinal electric potential induced by the application of a finite bias voltage, and the longitudinal electric potential is given by $V(x)=-e V_{0} \theta(x-L)$ $-e V_{0}(x / L) \theta(x) \theta(L-x)$, where $V_{0}$ is the magnitude of the applied bias voltage. Due to the application of the bias voltage $V_{0}$, a longitudinal electric field $F \equiv V_{0} / L$ will be established in the semiconductor channel of the structure and the Fermi energy $\mu_{R}$ in the right electrode will be lowered by $e V_{0}$ with respect to the Fermi energy $\mu_{L}$ in the left electrode.

To obtain the spin conductance of the device described by the Hamiltonian (1), we start by considering the scattering problem related to the interfaces between the $F$ and $S$ regions. In order to solve the scattering problem, one need to find first the eigenstates in each region. In the ferromagnetic electrodes $(x<0$ and $x>L)$, one obtains from the Hamiltonian (1) the eigenstates with energy $E$,

$$
\begin{aligned}
\Psi_{F, \sigma, L}^{( \pm)} & =\phi_{F, \sigma, L}^{( \pm)}(x)|\sigma\rangle, \phi_{F, \sigma, L}^{( \pm)}(x) \\
& =\sqrt{\frac{m_{f}}{\hbar k_{\sigma, L}}} e^{ \pm i k_{\sigma, L^{x}}}(x<0), \\
\Psi_{F, \gamma, R}^{( \pm)} & =\phi_{F, \gamma, R}^{( \pm)}(x)|\gamma\rangle, \phi_{F, \gamma, R}^{( \pm)}(x) \\
& =\sqrt{\frac{m_{f}}{\hbar k_{\gamma, R}}} e^{ \pm i k_{\gamma, R^{x}}}(x>L),
\end{aligned}
$$

where $|\sigma\rangle(\sigma= \pm)$ and $|\gamma\rangle(\gamma= \pm)$ are the spinor eigenstates in the left and right electrodes, respectively, which are defined by

$$
\left\{|+\rangle_{L},|-\rangle_{L}\right\}=\frac{1}{\sqrt{2}}\left(\begin{array}{l} 
\pm 1 \\
1
\end{array}\right),\left\{|+\rangle_{R},|-\rangle_{R}\right\}=\lambda \frac{1}{\sqrt{2}}\left(\begin{array}{l} 
\pm 1 \\
1
\end{array}\right),
$$

where $\lambda=+1$ if the two ferromagnetic electrodes are in parallel configuration and $\lambda=-1$ if the two ferromagnetic electrodes are in antiparallel configuration. The wave number $k_{\sigma, L}\left(k_{\gamma, R}\right)$ will be given by $k_{ \pm, L(R)}=\sqrt{\left(2 m_{f} / \hbar^{2}\right)(E \mp \Delta)}$. The eigenfunctions in the $S$ region cannot be written down directly from the Hamiltonian (1) due to the presence of the last term in Eq. (1). To find the eigenstates in the $S$ region, we first note that in the $S$ region the Hamiltonian (1) is spin diagonal and the eigenstates have the form $\Psi_{S, \beta}(x)$ $=\phi_{S, \beta}(x)|\beta\rangle$ and $\Psi_{S, \bar{\beta}}(x)=\phi_{S, \bar{\beta}}(x)|\bar{\beta}\rangle$, where $|\beta\rangle=(1,0)$ and $|\bar{\beta}\rangle=(0,1)$ are the spinor eigenstates in the $S$ region. The Schrödinger equation in the $S$ region will reduce to

$$
\begin{aligned}
- & \frac{\hbar^{2}}{2 m_{s}} \frac{\partial^{2}}{\partial x^{2}} \phi_{S, \beta}(x)-i \alpha_{R} \frac{\partial}{\partial x} \phi_{S, \beta}(x)-\frac{e V_{0} x}{L} \phi_{S, \beta}(x) \\
& =E \phi_{S, \beta}(x),
\end{aligned}
$$




$$
\begin{aligned}
- & \frac{\hbar^{2}}{2 m_{S}} \frac{\partial^{2}}{\partial x^{2}} \phi_{S, \bar{\beta}}(x)+i \alpha_{R} \frac{\partial}{\partial x} \phi_{S, \bar{\beta}}(x)-\frac{e V_{0} x}{L} \phi_{S, \bar{\beta}}(x) \\
& =E \phi_{S, \bar{\beta}}(x) .
\end{aligned}
$$

After making a transformation

$$
\phi_{S, \beta}(x) \rightarrow w_{\beta}(x)=\phi_{S, \beta}(x) e^{i \alpha_{R} m x / \hbar^{2}}
$$

and

$$
\phi_{S, \bar{\beta}}(x) \rightarrow w_{\bar{\beta}}(x)=\phi_{S, \bar{\beta}}(x) e^{-i \alpha_{R} m x / \hbar^{2}},
$$

it can be shown that both $w_{\beta}(x)$ and $w_{\bar{\beta}}(x)$ will satisfy the following equation:

$$
\frac{\partial^{2}}{\partial x^{2}} w(x)+\frac{2 e V_{0} m_{s}}{L \hbar^{2}}\left(x+\epsilon_{0}\right) w(x)=0,
$$

where $\epsilon_{0}$ is defined by

$$
\epsilon_{0}=\frac{E L}{e V_{0}}+\frac{\alpha_{R}^{2} m_{s} L}{2 e V_{0} \hbar^{2}} .
$$

Equation (7) can solved with the help of the Airy functions and the two linearly independent solutions can be given by $A i\left[-\left(2 e V_{0} m_{s} / L \hbar^{2}\right)^{1 / 3}\left(x+\epsilon_{0}\right)\right]$ and $B i[$ $\left.-\left(2 e V_{0} m_{s} / L \hbar^{2}\right)^{1 / 3}\left(x+\epsilon_{0}\right)\right]$. Here $A i[z]$ and $B i[z]$ are the usual Airy functions. ${ }^{29}$ Then one can see that in the $S$ region there are four independent eigenstates with energy $E$, and the corresponding eigenfunctions $\Psi_{S, \beta}^{(i)}(x)$ and $\Psi_{S, \bar{\beta}}^{(i)}(x) \quad(i$ $=1,2$ ) will be given by

$$
\begin{gathered}
\Psi_{S, \beta}^{(i)}(x)=\phi_{S, \beta}^{(i)}(x)|\beta\rangle, \phi_{S, \beta}^{(i)}(x)=e^{-i \alpha_{R} m x / \hbar^{2}} w^{(i)}(x), \\
\Psi_{S, \bar{\beta}}^{(i)}(x)=\phi_{S, \bar{\beta}}^{(i)}(x)|\bar{\beta}\rangle, \phi_{S, \bar{\beta}}^{(i)}(x)=e^{i \alpha_{R} m x / \hbar^{2}} w^{(i)}(x),
\end{gathered}
$$

where $\quad w^{(1)}(x) \equiv A i\left[-\left(2 e V_{0} m_{s} / L \hbar^{2}\right)^{1 / 3}\left(x+\epsilon_{0}\right)\right] \quad$ and $w^{(2)}(x) \equiv B i\left[-\left(2 e V_{0} m_{s} / L \hbar^{2}\right)^{1 / 3}\left(x+\epsilon_{0}\right)\right]$.

Now we consider the scattering state of an electron with energy $E$ and spin $\sigma$ incoming from the ferromagnetic lead $(x<0)$. The total wave function including the reflected and transmitted waves can be written as

$$
\begin{gathered}
\Psi_{F}(x)=\phi_{F, \sigma, L}^{(+)}(x)|\sigma\rangle+r_{\sigma \sigma} \phi_{F, \sigma, L}^{(-)}(x)|\sigma\rangle \\
+r_{\sigma \bar{\sigma}} \phi_{F, \bar{\sigma}, L}^{(-)}(x)|\bar{\sigma}\rangle, \quad x<0, \\
\Psi_{S}(x)=\sum_{i=1,2} c_{i, \beta} \phi_{S, \beta}^{(i)}(x)|\beta\rangle \\
+\sum_{i=1,2} c_{i, \bar{\beta}} \phi_{S, \bar{\beta}}^{(i)}(x)|\bar{\beta}\rangle, \quad 0<x<L, \\
\Psi_{F}(x)=t_{\sigma \gamma} \phi_{F, \gamma, R}^{(+)}(x)|\gamma\rangle+t_{\sigma \bar{\gamma}} \phi_{F, \bar{\gamma}, R}^{(+)}(x)|\bar{\gamma}\rangle, \quad x>L,
\end{gathered}
$$

where $r_{\sigma \sigma}, r_{\sigma \bar{\sigma}}, t_{\sigma \gamma}, t_{\sigma \bar{\gamma}}, c_{i, \beta}$, and $c_{i, \bar{\beta}}(i=1,2)$ are coefficients that need to be determined by the boundary condi- tions. The matching conditions at the interfaces between the $F$ and $S$ regions can be obtained by integrating $\hat{H} \Psi=E \Psi$ from $x=-\varepsilon$ to $x=+\varepsilon$ and from $x=L-\varepsilon$ to $x=L+\varepsilon$ in the limit $\varepsilon \rightarrow 0$. This yields

$$
\begin{gathered}
\left.\Psi_{F}(x)\right|_{x=0^{-}}=\left.\Psi_{S}(x)\right|_{x=0^{+}}, \\
\left.\Psi_{S}(x)\right|_{x=L^{-}}=\left.\Psi_{F}(x)\right|_{x=L^{+}}, \\
\left.\hat{v}_{S} \Psi_{S}(x)\right|_{x=0^{+}}=\left.\hat{v}_{F} \Psi_{F}(x)\right|_{x=0^{-}}-\left.\frac{2 i}{\hbar} \hat{U} \Psi_{F}(x)\right|_{x=0^{-}}, \\
\left.\hat{v}_{S} \Psi_{S}(x)\right|_{x=L^{-}}=\left.\hat{v}_{F} \Psi_{F}(x)\right|_{x=L^{+}}+\left.\frac{2 i}{\hbar} \hat{U} \Psi_{F}(x)\right|_{x=L^{+}},
\end{gathered}
$$

where $\hat{v}_{F}=\hat{p}_{x} / m_{f}$ and $\hat{v}_{S}=\hat{p}_{x} / m_{s}+\left(\alpha_{R} / \hbar\right) \hat{\sigma}_{z}$ are the velocity operators in the $F$ and $S$ regions, respectively. From the matching conditions (14)-(17), the transmission coefficients $t_{\sigma \gamma}$ and $t_{\sigma \bar{\gamma}}$ can be obtained. Then in the linear-response regime and in the low-temperature limit, the spin conductance $G_{\sigma}$ and the total conductance $G$ of the device can be calculated through the Landauer formula, given by

$$
G=\sum_{\sigma= \pm} G_{\sigma}, G_{\sigma}=\frac{e^{2}}{h} \sum_{\gamma= \pm}\left|t_{\sigma \gamma}(\mu)\right|^{2},
$$

where $\mu$ is the average of the Fermi energies $\mu_{L}$ and $\mu_{R}$ on the left and right electrodes, respectively. ${ }^{30}$ The spin injection coefficient for the device can be defined by $\eta=\left(G_{+}\right.$ $\left.-G_{-}\right) /\left(G_{+}+G_{+}\right)$. This ratio characterizes the spin polarization of the charge current flowing through the device. The conductance of the device and the spin injection coefficient will depend on the magnetization configurations in the two electrodes. In the following we will denote the conductance as $G^{(P)}$ and the spin injection coefficient as $\eta^{(P)}$ if the magnetizations in the two electrodes are parallel and as $G^{(A P)}$ and $\eta^{(A P)}$ if the magnetizations in the two electrodes are antiparallel. The change in conductance when the two ferromagnetic electrodes switch between parallel and antiparallel configurations can be measured by a magnetoconductance ratio $\eta_{M}$, defined by

$$
\eta_{M}=\frac{G^{(P)}-G^{(A P)}}{G^{(P)}+G^{(A P)}} .
$$

\section{RESULTS AND DISCUSSIONS}

Based on the formulas established above, in this section we will present some numerical examples by considering some actual experimental parameters. We will solve Eqs. (14)-(17) numerically by transfer-matrix method. In order to obtain the transfer matrix, it may be more convenient to rewrite the wave function in the electrodes in a more general form as following:

$$
\begin{aligned}
\Psi_{F}(x)= & \sum_{\sigma= \pm}\left[a_{\sigma}^{(+)} \phi_{F, \sigma, L}^{(+)}(x)|\sigma\rangle+a_{\sigma}^{(-)} \phi_{F, \sigma, L}^{(-)}(x)|\sigma\rangle\right], \\
& x<0
\end{aligned}
$$



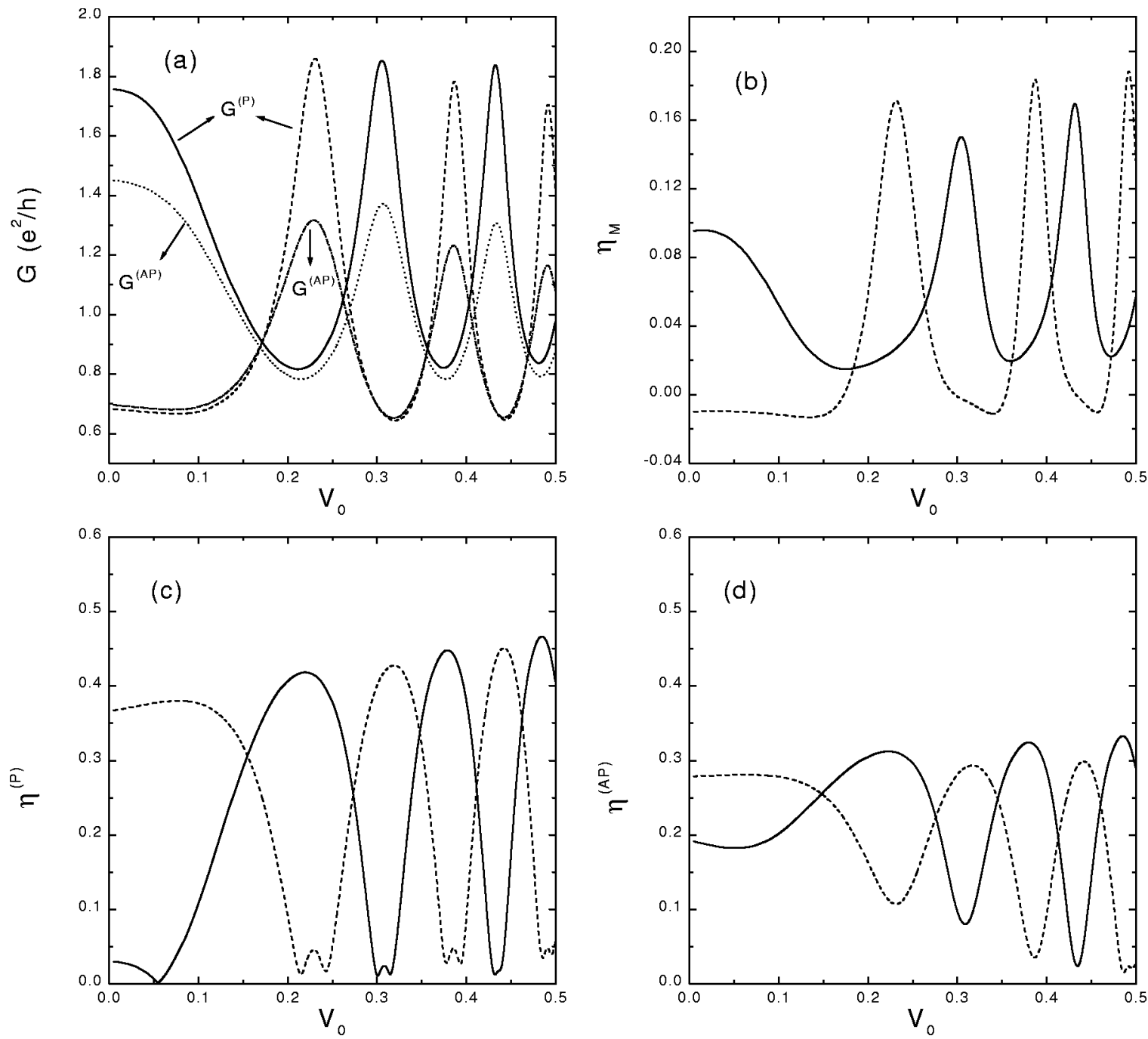

FIG. 1. The changes of the conductance $G^{(P)}$ and $G^{(A P)}$, the magnetoconductance ratio $\eta_{M}$, and the spin injection coefficient $\eta^{(P)}$ and $\eta^{(A P)}$, with the variations of the bias voltage $V_{0}$ in two distinct cases with different Rashba spin-orbit coupling constant. [The strength of Rashba spin-orbit coupling is characterized by the Rashba wave number $k_{R} \equiv m_{s} \alpha_{R} / \hbar^{2}$. In Fig. 1(a), $k_{R}=10^{7} \mathrm{~cm}^{-1}$ for the solid line and the dotted line, $k_{R}=5 \times 10^{7} \mathrm{~cm}^{-1}$ for the dashed line and the dash-dotted line. In (b)-(d), $k_{R}=10^{7} \mathrm{~cm}^{-1}$ for the solid line and $k_{R}=5$ $\times 10^{7} \mathrm{~cm}^{-1}$ for dashed line. Other parameters were given in the text.]

$$
\begin{aligned}
\Psi_{F}(x)= & \sum_{\gamma= \pm}\left[b_{\gamma}^{(+)} \phi_{F, \gamma, R}^{(+)}(x)|\gamma\rangle+b_{\gamma}^{(-)} \phi_{F, \gamma, R}^{(-)}(x)|\gamma\rangle\right], \\
& x>L .
\end{aligned}
$$

If the spin of incident electron is $|\sigma\rangle$, one has $a_{\sigma}^{(+)}=1$, $a_{\bar{\sigma}}^{(+)}=0, \quad a_{\sigma}^{(-)}=r_{\sigma \sigma}, \quad a_{\bar{\sigma}}^{(-)}=r_{\sigma \bar{\sigma}}, \quad b_{\gamma}^{(+)}=t_{\sigma \gamma}, \quad b_{\bar{\gamma}}^{(+)}=t_{\sigma \bar{\gamma}}$, $b_{\gamma}^{(-)}(\gamma= \pm)$ will be set to be zero. From Eq. (12) and Eqs. (20)-(21), at the interfaces between the $F$ and $S$ regions, $\Psi_{F}(x), \Psi_{S}(x), \hat{v}_{F} \Psi_{F}(x)$, and $\hat{v}_{S} \Psi_{S}(x)$ can be expressed as following:

$$
\begin{gathered}
{\left[\begin{array}{l}
\left.\Psi_{S}(x)\right|_{x=0^{+}} \\
\left.\hat{v}_{S} \Psi_{S}(x)\right|_{x=0^{+}}
\end{array}\right]=\hat{S}_{2}\left[\begin{array}{c}
c_{1, \beta} \\
c_{2, \beta} \\
c_{1, \bar{\beta}} \\
c_{2, \bar{\beta}}
\end{array}\right],} \\
{\left[\begin{array}{l}
\left.\Psi_{S}(x)\right|_{x=L^{-}} \\
\left.\hat{v}_{S} \Psi_{S}(x)\right|_{x=L^{-}}
\end{array}\right]=\hat{S}_{3}\left[\begin{array}{c}
c_{1, \beta} \\
c_{2, \beta} \\
c_{1, \bar{\beta}} \\
c_{2, \bar{\beta}}
\end{array}\right],} \\
{\left[\begin{array}{l}
\Psi_{F}(x)_{x=L^{+}} \\
\left.\hat{v}_{F} \Psi_{F}(x)\right|_{x=L^{+}} ^{(+)}
\end{array}\right]=\hat{S}_{4}\left[\begin{array}{c}
b_{-}^{(+)} \\
b_{+}^{(-)} \\
b_{-}^{(-)}
\end{array}\right],}
\end{gathered}
$$$$
\left[\begin{array}{l}
\left.\Psi_{F}(x)\right|_{x=0^{-}} \\
\left.\hat{v}_{F} \Psi_{F}(x)\right|_{x=0^{-}}
\end{array}\right]=\hat{S}_{1}\left[\begin{array}{c}
a_{+}^{(+)} \\
a_{-}^{(+)} \\
a_{+}^{(-)} \\
a_{-}^{(-)}
\end{array}\right],
$$ 

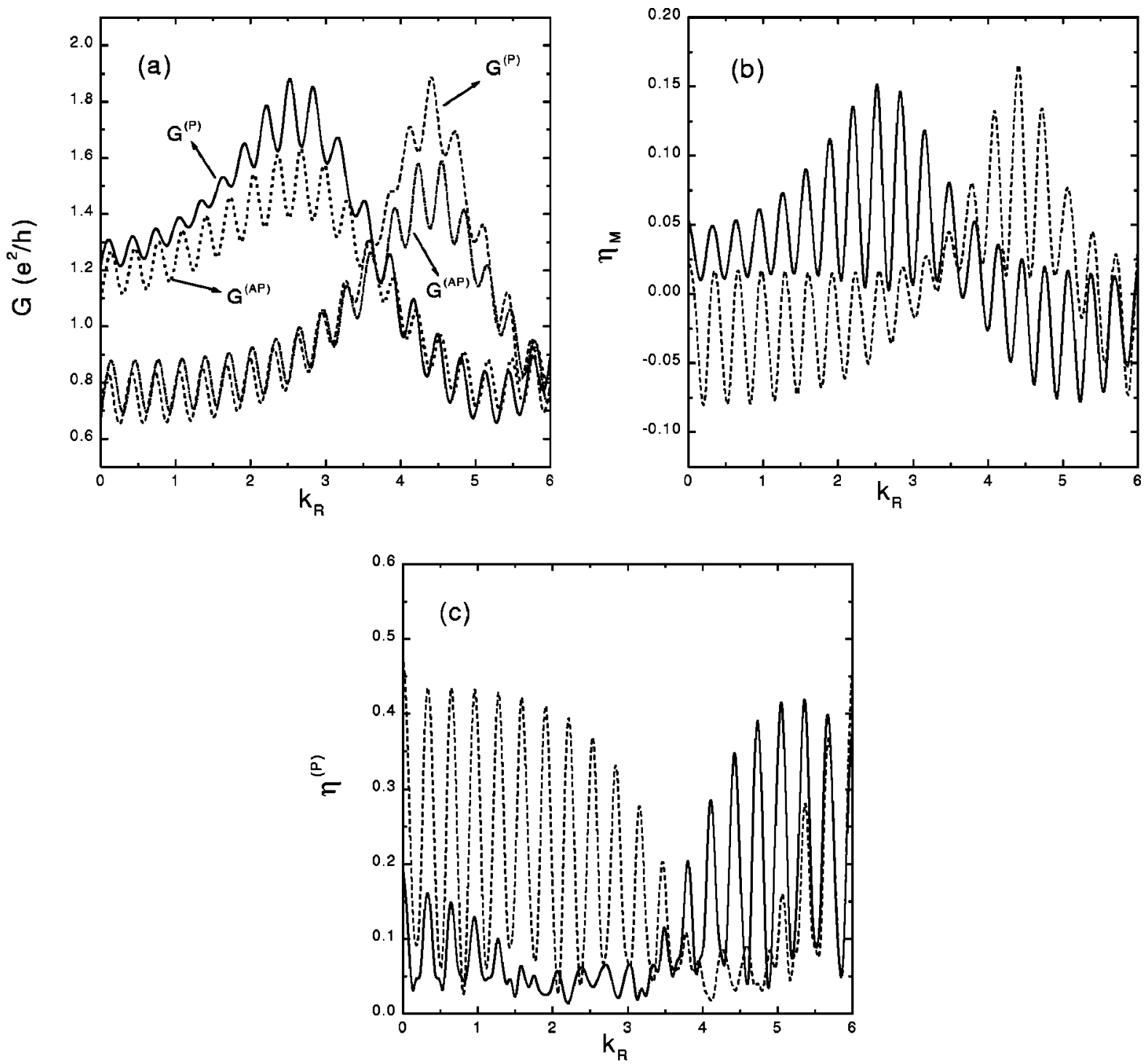

FIG. 2. The changes of the conductance $G^{(P)}$ and $G^{(A P)}$, the magnetoconductance ratio $\eta_{M}$, and the spin injection coefficient $\eta^{(P)}$, with the variations of the Rashba wave number $k_{R}$ in two distinct cases with different bias voltages. [In (a), $V_{0}=0.1 \mathrm{~V}$ for the solid line and the dotted line, $V_{0}=0.2 \mathrm{~V}$ for the dashed line and the dash-dotted line. In (b) $-(\mathrm{c}), V_{0}=0.1 \mathrm{~V}$ for the solid line and $V_{0}=0.2 \mathrm{~V}$ for the dashed line. Other parameters were given in the text. The changes of the spin injection coefficient $\eta^{(A P)}$ with the variations of $k_{R}$ is similar as was shown in (c) and were not plotted.]

where $\hat{S}_{i}(i=1,2,3,4)$ are $4 \times 4$ matrices, and the matrix elements of $\hat{S}_{i}$ can be written down directly from Eqs. (12) and Eqs. (20)-(21). From the matching condition (14)-(17) and Eq. (22), one gets that

$$
\left[\begin{array}{c}
a_{+}^{(+)} \\
a_{-}^{(+)} \\
a_{+}^{(-)} \\
a_{-}^{(-)}
\end{array}\right]=\hat{S}_{t}\left[\begin{array}{c}
b_{+}^{(+)} \\
b_{-}^{(+)} \\
b_{+}^{(-)} \\
b_{-}^{(-)}
\end{array}\right],
$$

where $\hat{S}_{t} \equiv \hat{S}_{1}^{-1} \hat{S}_{2} \hat{S}_{3}^{-1} \hat{S}_{4}$ are the transfer matrix. Taking $b_{+}^{(-)}=0$ and $b_{-}^{(-)}=0$, then from Eq. (23) one gets that

$$
\left[\begin{array}{c}
b_{+}^{(+)} \\
b_{-}^{(+)}
\end{array}\right]=\hat{T}\left[\begin{array}{l}
a_{+}^{(+)} \\
a_{-}^{(+)}
\end{array}\right], \hat{T}=\left[\begin{array}{ll}
S_{t}(1,1) & S_{t}(1,2) \\
S_{t}(2,1) & S_{t}(2,2)
\end{array}\right]^{-1}
$$

where $S_{t}(i, j)$ are the matrix elements of the transfer matrix $\hat{S}_{t}$. Since $a_{\sigma}^{(+)}=1$ and $a_{\sigma}^{(+)}=0$ if the spin of incident electron is $|\sigma\rangle$, then the transmission coefficient can be obtained directly from Eq. (24) as following: $t_{++}=T(1,1), t_{+-}$ $=T(2,1), t_{-+}=T(1,2), t_{--}=T(2,2)$, where $T(i, j)$ are the elements of the matrix $\hat{T}$. After the transmission coefficients are obtained, the spin conductance of the device can be obtained from Eq. (18). In the following we will focus on iron $(\mathrm{Fe})$ as the ferromagnetic source and drain and InAs as the semiconductor channel. In the ferromagnetic electrodes the Fermi energy (in the equilibrium state) will be set to $E_{F}$ $=2.469 \mathrm{eV}$ and the exchange splitting energy be set to $\Delta$ 

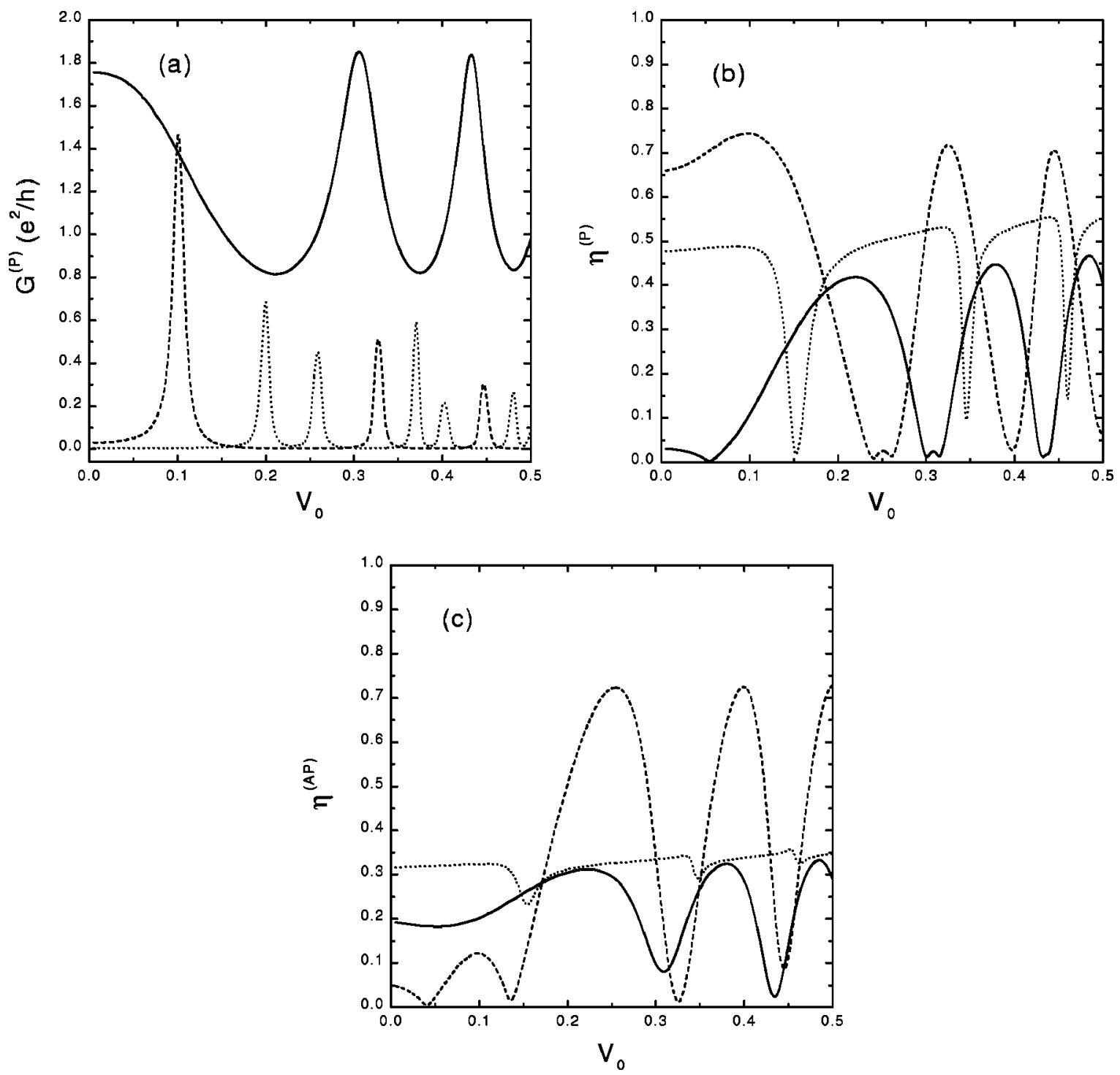

FIG. 3. The changes of the conductance $G^{(P)}$ and the spin injection coefficient $\eta^{(P)}$ and $\eta^{(A P)}$ with the variations of the bias voltage $V_{0}$ in the presence of interfacial scattering. [The strengths of interfacial scattering are characterized by two dimensionless parameters $z_{1}$ and $z_{2}$. In (a), $z_{1}=0$ and $z_{2}=0$ for the solid line; $z_{1}=10$ and $z_{2}=0$ for the dashed; $z_{1}=0$ and $z_{2}=10$ for the dotted line. In (b) $-(\mathrm{c}), z_{1}=0$ and $z_{2}=0$ for the solid line; $z_{1}=5$ and $z_{2}=0$ for the dotted line; $z_{1}=0$ and $z_{2}=5$ for the dashed line. $k_{R}=10^{7} \mathrm{~cm}^{-1}$. Other parameters were given in the text. The changes of the conductance $G^{(A P)}$ with the variations of the bias voltage $V_{0}$ is similar as was shown in (a) and were not plotted.]

$=3.46 \mathrm{eV}$, appropriate for Fe. The effective masses were set to $m_{f}=m_{e}$ (for $\mathrm{Fe}$ ) and $m_{s}=0.036 m_{e}$ (for InAs), and the band mismatch between the $F$ and $S$ regions were set to $\delta E_{c}=2.0 \mathrm{eV}$. The length of the semiconductor channel was set to be $1 \mu \mathrm{m}$. The strength of Rashba spin-orbit coupling will be characterized by a Rashba wave number $k_{R}$ $\equiv m_{s} \alpha_{R} / \hbar^{2}$. For simplicity, we first assume that no interfacial scattering presents (i.e., $\hat{U}=0$ ). In Figs. 1(a)-1(b) we have plotted the changes of the total conductance $G^{(P)}$ and $G^{(A P)}$ and the magnetoconductance ratio $\eta_{M}$ with the variations of the bias voltage $V_{0}$ in two distinct cases with different Rashba spin-orbit coupling constants, and the changes of the spin injection coefficient $\eta^{(P)}$ and $\eta^{(A P)}$ with the variations of the bias voltage $V_{0}$ were also plotted in Figs. 1(c)1(d), respectively. From Figs. 1(a)-1(d) one can see that in a large range of the bias voltage $V_{0}$, the conductance and the magnetoconductance ratio and the spin injection coefficient all can be changed significantly by tuning the Rashba spinorbit coupling (which can be realized by changing the gate voltage), suggesting that the structure described the Hamiltonian (1) may exhibit significant spin-transistor effect in a large range of the bias voltage. But Figs. 1(a)-1(d) show that the modulations of the conductance and the magnetoconductance ratio and the spin injection coefficient due to the Rashba spin-orbit coupling may depend sensitively on the bias voltage, i.e., the changes of the conductance and the magnetoconductance ratio and the spin injection coefficient with the variations of the Rashba spin-orbit coupling constant may be very different under different bias voltages. This can be seen more clearly from Figs. 2(a)-2(c), where we have plotted the changes of the conductance $G^{(P)}$ and $G^{(A P)}$ and the magnetoconductance ratio $\eta_{M}$ and the spin 
injection coefficient $\eta^{(P)}$ with the variations of the Rashba spin-orbit coupling constant (characterized by the Rashba wave number $k_{R} \equiv m_{s} \alpha_{R} / \hbar^{2}$ ) in two distinct cases with different bias voltage $V_{0}$. From Figs. 2(a)-2(c) one can see clearly that the bias voltage may have significant influence on the modulations of the conductance and the magnetoconductance ratio and the spin injection coefficient due to Rashba spin-orbit coupling. From theoretical viewpoints, the spin-transistor effect due to Rashba spin-orbit coupling may depend sensitively on the bias voltage because that the application of a finite bias voltage will not only change the energies of incident electrons (as in usual electronic devices) but also have influence on the gate-voltage controlled spin precession of injected spin-polarized electrons in the $S$ channel of the device. The reason for this is that when a finite bias voltage is applied between two terminals of a spin FET, a longitudinal electric field will be established in the $S$ channel of the device, and due to the presence of this longitudinal electric field, spin precession of injected spin-polarized electrons in the $S$ channel will depend not only on the gatevoltage controlled Rashba spin-orbit coupling but also depend on the bias voltage. This can be seen clearly from the formulas presented in Sec. II, where we have shown that in the presence of a finite bias voltage, the spinor wave function in the $S$ region will depend not only on the Rashba spin-orbit coupling but also on the bias voltage. So, in order to describe correctly the spin precession states of injected spin-polarized electrons in the semiconductor channel of a spin FET, the interplay of the gate-voltage controlled Rashba spin-orbit coupling and the longitudinal electric field induced by the application of a finite bias voltage need to be described in a unified way, as was shown in Sec. II. Next, we consider the effect of interfacial scattering (i.e., $\hat{U} \neq 0$ ). The strength of interfacial scattering can be characterized by two dimensionless parameters defined by $z_{1} \equiv\left(U_{1} / \hbar\right) \sqrt{2 m_{f} / E_{F}}$ and $z_{2}$ $\equiv\left(U_{2} / \hbar\right) \sqrt{2 m_{f} / E_{F}}$, where $U_{1}$ and $U_{2}$ are the diagonal and off-diagonal elements of the interfacial scattering potential matrix $\hat{U}$. The parameters $z_{1}$ and $z_{2}$ represent the strengths of spin-conserving and spin-flip interfacial scatterings, respectively. The effect of interfacial scatterings can be seen from Figs. 3(a)-3(c), where we have plotted the changes of the conductance $G^{(P)}$ and the spin injection coefficient $\eta^{(P)}$ and $\eta^{(A P)}$ with the variations of the bias voltage $V_{0}$ in the presence of interfacial scatterings. Figure 3(a) shows that both spin-conserving and spin-flip interfacial scatterings will decrease substantially the conductance of the device, but Figs. 3(b)-3(c) show that the effect of interfacial scatterings on the spin injection coefficient is very different from its effect on the conductance. From Figs. 3(b)-3(c) one can see that both spin-conserving and spin-flip interfacial scattering can enhance rather than decrease the spin injection efficiency across the $F / S$ interfaces in the device. This is a little similar as in the corresponding case of spin injections in diffusive FSF junctions, where one can show that if the contact between the $F$ and $S$ regions are both resistive and spin-selective, the spin injection efficiency across the FSF junctions can be increased substantially and the problem of conductivity mismatch can be remedied. ${ }^{9}$ From theoretical viewpoints, this enhancement arises from the fact that when moving electrons are transmitted between the $F$ regions and the $S$ regions in a FSF junction, spin-up and spin-down electrons will experience asymmetric scattering, and this asymmetry can be enhanced in the presence of spin-dependent interfacial scatterings.

In conclusion, in this paper we have discussed the influence of finite bias voltages on the conductance modulations in spin FET's due to Rashba spin-orbit coupling. We have shown that when a finite bias voltage is applied between two terminals of a spin FET, the conductance modulation in the device due to Rashba spin-orbit coupling may depend sensitively on the bias voltage, and the spin precession states of injected spin-polarized electrons in the semiconductor channel of the device will depend not only on the gate-voltage controlled Rashba spin-orbit coupling but also on the bias voltage. In the approach presented in this paper, the effect of the interplay of the Rashba spin-orbit coupling and the bias voltage have been described in a unified way.

\section{ACKNOWLEDGMENT}

This work was supported by a grant from the Research Grant Council of Hong Kong, China.
${ }^{1}$ S.A. Wolf, D.D. Awschalom, R.A. Buhrman, J.M. Daughton, S. Von Molnar, M.L. Roukes, A.Y. Chtchelkanova, and D.M. Treger, Science 294, 1488 (2001); P. Ball, Nature (London) 404, 918 (2000).

${ }^{2}$ G.A. Prinz, Science 282, 1660 (1998); Phys. Today 48 (4), 58 (1995).

${ }^{3}$ J.M. Kikkawa, Phys. Today 52 (6), 33 (1999).

${ }^{4}$ S. Datta and B. Das, Appl. Phys. Lett. 56, 665 (1990).

${ }^{5}$ S.-Q. Shen and X.C. Xie, Phys. Rev. B 67, 144423 (2003); S.-Q. Shen, Z.J. Li, and Z.S. Ma, Appl. Phys. Lett. 84, 996 (2004).

${ }^{6}$ Y. Ohno, D.K. Young, B. Beschoten, F. Matsukura, H. Ohno, and D.D. Awschalom, Nature (London) 402, 790 (1999); R. Fiederling, M. Keim, G. Reuscher, W. Ossau, G. Schmidt, A. Waag, and L.W. Molenkamp, ibid. 402, 787 (1999).
${ }^{7}$ G. Schmidt, D. Ferrand, L.W. Molenkamp, A.T. Filip, and B.J. van Wees, Phys. Rev. B 62, R4790 (2000).

${ }^{8}$ M. Johnson and R.H. Silsbee, Phys. Rev. B 35, 4959 (1987).

${ }^{9}$ E.I. Rashba, Phys. Rev. B 62, R16267 (2000); Eur. Phys. J. B 29, 513 (2002).

${ }^{10}$ A. Fert and H. Jaffres, Phys. Rev. B 64, 184420 (2001).

${ }^{11}$ G. Kirczenow, Phys. Rev. B 63, 054422 (2001).

${ }^{12}$ P. Mavropoulos, O. Wunnicke, and H. Dederichs, Phys. Rev. B 66, 024416 (2002).

${ }^{13}$ V.P. LaBella, D.W. Bullock, Z. Ding, C. Emery, A. Venkatesan, W.F. Oliver, G.J. Salamo, P.M. Thibado, and M. Mortazavi, Science 292, 1518 (2001).

${ }^{14}$ H.J. Zhu, M. Ramsteiner, H. Kostial, M. Wassermeier, H.-P. Schönherr, and K.H. Ploog, Phys. Rev. Lett. 87, 016601 (2001). 
${ }^{15}$ A.T. Hanbicki and B.T. Jonker, Appl. Phys. Lett. 80, 1240 (2002).

${ }^{16}$ G. Lommer, F. Malcher, and U. Rössler, Phys. Rev. Lett. 60, 728 (1988).

${ }^{17}$ Y.A. Bychkov and E.I. Rashba, J. Phys. C 17, 6039 (1984).

${ }^{18}$ J. Luo, H. Munekata, F.F. Fang, and P.J. Stiles, Phys. Rev. B 38, 10142 (1988).

${ }^{19}$ B. Das, D.C. Miller, S. Datta, R. Reifenberger, W.P. Hong, P.K. Bhattacharya, J. Singh, and M. Jaffe, Phys. Rev. B 39, 1411 (1989).

${ }^{20}$ D. Grundler, Phys. Rev. B 63, R161307 (2001).

${ }^{21}$ C.M. -Hu and T. Matsuyama, Phys. Rev. Lett. 87, 066803 (2001); T. Matsuyama, C.M. -Hu, D. Grundler, G. Meier, and U. Merkt, Phys. Rev. B 65, 155322 (2002).

${ }^{22}$ M.H. Larsen, A.M. Lunde, and K. Flensberg, Phys. Rev. B 66, 033304 (2002).

${ }^{23}$ L.W. Molenkamp, G. Schmidt, and G.E.W. Bauer, Phys. Rev. B
64, 121202(R) (2001).

${ }^{24}$ F. Mireles and G. Kirczenow, Phys. Rev. B 64, 024426 (2001); Europhys. Lett. 59, 107 (2002).

${ }^{25}$ J. Schliemann, J.C. Egues, and D. Loss, Phys. Rev. Lett. 90, 146801 (2003).

${ }^{26}$ J.E. Hirsch, Phys. Rev. Lett. 83, 1834 (1999).

${ }^{27}$ S. Murakami, N. Nagaosa, and S.-C. Zhang, Science 301, 1348 (2003)

${ }^{28}$ L.B. Hu, J. Gao, and S.-Q. Shen, Phys. Rev. B 68, 115302 (2003); 68, 153303 (2003).

${ }^{29}$ M. Abramowitz and I.A. Stegun, Handbook of Mathematical Functions (Dover, New York, 1965).

${ }^{30}$ Mesoscopic Physics and Electronics, edited by T. Ando, Y. Arakawa, K. Furuya, S. Komiyama, and H. Nakashima (Springer, New York, 1998). 